\title{
Is quetiapine suitable for treatment of acute schizophrenia with catatonic stupor? A case series of 39 patients
}

This article was published in the following Dove Press journal:

Neuropsychiatric Disease and Treatment

9 October 2013

Number of times this article has been viewed

\author{
Bunta Yoshimura ${ }^{1,2}$ \\ Tomoya Hirota ${ }^{3}$ \\ Manabu Takaki \\ Yoshiki Kishi' \\ 'Department of Psychiatry, Okayama \\ Psychiatric Medical Center, \\ ${ }^{2}$ Department of Neuropsychiatry, \\ Okayama University Graduate \\ School of Medicine, Dentistry and \\ Pharmaceutical Sciences, Okayama, \\ Japan; ${ }^{3}$ Department of Psychiatry, \\ Vanderbilt University Medical Center, \\ Nashville, TN, USA
}

Purpose: We aimed to determine which antipsychotic is most effective for the treatment of acute schizophrenia with catatonic stupor.

Patients and methods: Data were obtained from the medical records of 450 patients with the diagnosis of schizophrenia, who had received acute psychiatric inpatient treatment between January 2008 and December 2010 at our hospital. Among them, 39 patients $(8.7 \%)$ met the definition of catatonic stupor during hospitalization. The diagnoses of schizophrenia in all 39 patients were reconfirmed during the maintenance phase. We retrospectively reviewed the medical records of these 39 patients to investigate which antipsychotics were chosen for treatment during the period from admission to recovery from catatonia, at the time of discharge, and 12 and 30 months after discharge.

Results: As compared to other antipsychotics, it was found out that use of quetiapine had better outcomes and hence was used more often. A total of $61.5 \%$ of patients were on quetiapine at the time of recovery from catatonia and $51.3 \%$ of patients were on quetiapine at the time of discharge as compared to only $17.9 \%$ of patients on quetiapine on admission. However, at 12 and 30 months after discharge, the rates had decreased to $38.4 \%$ and $25.6 \%$. Similarly, of 29 patients who were not administered electroconvulsive therapy, quetiapine was used at significantly higher rates at the time of recovery from catatonia $(48.3 \%)$ than at the time of admission (17.2\%). All 39 patients had received an antipsychotic as the first-line treatment and some antipsychotics might have contributed to the development of catatonia.

Conclusion: This study suggests that quetiapine is a promising agent for the treatment of schizophrenia with catatonic stupor during the acute phase.

Keywords: catatonia, electroconvulsive therapy, quetiapine, schizophrenia, stupor

\section{Introduction}

Catatonia is a syndrome characterized by marked psychomotor abnormalities associated with schizophrenia, mood disorders, general medical conditions, drug withdrawal, and toxic drug states. ${ }^{1}$ The clinical symptoms of catatonia are stupor, catalepsy, excessive and purposeless motor activity, extreme negativism, mutism, peculiarities of voluntary movement, echolalia, and echopraxia. ${ }^{2,3}$ Catatonia is observed in $5 \%-15 \%$ of psychiatric patients who require acute psychiatric hospital treatment. The predilection of psychiatrists to diagnose catatonic patients with schizophrenia appears to be based on historical reasons. ${ }^{4}$ However, previous studies show that most cases of catatonia are caused by mood disorders, irrespective of the age group, while $10 \%-20 \%$ of them are caused by schizophrenia. ${ }^{5-7}$
Correspondence: Bunta Yoshimura Okayama Psychiatric Medical Center, 3-16 Shikatahonmachi, Kita-ku, Okayama City, 700-0915, Japan

Tel +8I 86225382 I

Fax +8I 862252324

Email hajime-greentea@hotmail.co.jp (c) (i) (5) 2013 Yoshimura et al. This work is published by Dove Medical Press Limited, and licensed under Creative Commons Attribution - Non Commercial (unported, v3.0) License. The full terms of the License are available at http://creativecommons.org/licenses/by-nc/3.0/. Non-commercial uses of the work are permitted without any further permission from Dove Medical Press Limited, provided the work is properly attributed. Permissions beyond the scope of the License are administered by Dove Medical Press Limited. Intormation on
how to request permission may be found at: http://www.dovepress.com/permissions.php 
Malignant catatonia is a condition in which psychomotor symptoms of catatonia are accompanied by autonomic instability or hyperthermia. ${ }^{8}$ This frequently fatal condition cannot be distinguished from neuroleptic malignant syndrome (NMS) either clinically or by laboratory tests, which leads to the conclusion that NMS is a variant form of malignant catatonia, and that treating catatonia with antipsychotics is recognized as a risk factor for the development of NMS. ${ }^{9-11}$

Benzodiazepines (BZP) and electroconvulsive therapy (ECT) are the only recognized treatments of catatonia., ${ }^{5}-16$ Numerous articles indicate that BZP are rapidly effective, safe, and easily administered and are therefore regarded as suitable for first-line treatment for catatonia. If catatonic symptoms do not resolve rapidly with BZP, ECT is indicated. It may be necessary in some cases to treat with antipsychotics, but some authors recommend that antipsychotics should be avoided altogether in patients with catatonia because their use may be harmful. ${ }^{6,17}$

The number of cases of catatonia due to schizophrenia is minor, but the treatment of acute schizophrenia with catatonia is very complex, and the use of antipsychotics in patients with catatonia is controversial. ${ }^{6}$ Catatonic symptoms of patients with schizophrenia are somewhat less likely to respond to BZP. ${ }^{12,18}$ Even if catatonic symptoms are resolved rapidly with BZP, they may recur without adequate treatment of the underlying cause. ${ }^{5}$ However, which antipsychotic is most effective for the treatment of acute schizophrenia with catatonia has not been determined. To our knowledge, there is no more suggestive indication for treating schizophrenia with catatonia than the case reports of successful treatment with aripiprazole, ${ }^{19,20}$ risperidone, ${ }^{21,22}$ olanzapine, ${ }^{23,24}$ ziprasidone, ${ }^{25}$ and clozapine. ${ }^{26}$ In particular, catatonic stupor states have considerable potential for serious complications, which include dehydration, venous thrombosis, pulmonary embolism, and pneumonia. To determine the most effective antipsychotic for the treatment of acute schizophrenia with catatonic stupor, we reviewed the medical records of 39 patients with the diagnosis of schizophrenia with catatonic stupor.

\section{Methods}

The sample data was obtained from the medical records of 450 patients with the diagnosis of schizophrenia, not including schizoaffective disorder, based on the Diagnostic and Statistical Manual of Mental Disorders, fourth edition (DSM-IV), ${ }^{2}$ who had received acute psychiatric inpatient treatment between January 2008 and December 2010 on the acute psychiatric ward of the Okayama Psychiatric Medical Center. Among them, with the exclusion of patients with catatonic stupor associated with general medical conditions, drug withdrawal, and toxic drug states, 39 patients (8.7\%) met the definition of schizophrenia with catatonic stupor during hospitalization. All patients had formal medical evaluations by an internist to rule out underlying medical conditions that might have contributed to their psychiatric symptoms. The diagnoses of schizophrenia in all 39 patients were reconfirmed during the maintenance phase through clinical interview by each attending physician.

Because our hospital had no specific treatment algorithm, patients with schizophrenia characterized by catatonia were merely treated with antipsychotics in the same way as those without catatonia at the discretion of the attending physician.

Retrospectively, we reviewed the medical records of 39 patients with the diagnosis of schizophrenia with catatonic stupor to investigate which antipsychotics were used for treatment during the period from admission to recovery from catatonia, at the time of discharge, and 12 and 30 months after discharge. We defined "recovery from catatonia" as a mental state without catatonic symptoms or recurrence of catatonia during hospitalization. All 39 patients had made a "recovery from catatonia" prior to discharge. To assess the severity, we routinely used the Bush-Francis Catatonia Rating Scale ${ }^{3}$ and Clinical Global Impression-Severity scale in the acute and maintenance phases. We also investigated the presence of fever $\left(>38^{\circ} \mathrm{C}\right)$, high serum creatine kinase (h-CK; $\left.>500 \mathrm{IU} / \mathrm{L}\right)$, NMS (on the basis of the criteria for NMS of Caroff and Mann), ${ }^{27}$ duration of catatonia, other pharmacotherapies, and co-treatment with ECT. We classified the condition of patients with stupor in the presence of both fever and h-CK as "severe," and defined the others as "less severe."

The Institutional Review Board of our hospital approved this study. Because data for this study were collected in the course of routine clinical care, and these data were analyzed retrospectively and anonymized, informed consent was neither sought nor obtained.

Statistical analysis was conducted using SPSS Statistics 19 (IBM Corporation, Armonk, NY, USA). Continuous variables were analyzed using the independent sample $t$-test; categorical variables were analyzed using the chi-square test. The level of significance was set at $P<0.05$.

\section{Results}

Of 39 patients with catatonic stupor, 28 and 11 patients met the criteria of less severe and severe stupor, respectively (Table 1). 
Figure 1 is a flow chart of antipsychotics used for the 39 patients during the period from admission to recovery from catatonia.

Thirty-two patients (82.1\%) had an established diagnosis of schizophrenia prior to presenting with catatonia. The mean duration of untreated psychosis of eleven patients with first-episode was $31.0 \pm 46.2$ weeks. The mean time interval between the initial diagnosis of schizophrenia and the time-point of presentation with catatonia of 28 patients with multi-episode was $13.8 \pm 10.4$ years.

The frequency of catatonic symptoms, in order of declining frequency, were as follows: immobility/stupor, mutism and staring, 39/39; posturing/catalepsy, 36/39; mannerism, $30 / 39$; rigidity and withdrawal, 26/39; grimacing, 24/39; autonomic abnormality, 22/39; negativism, 20/39; excitement, 19/39; impulsivity, 14/39; gegenhalten, 10/39; verbigeration, 9/39; stereotypy, 7/39; waxy flexibility, 5/39; ambitendency, 4/39; automatic obedience and combativeness, 2/39; echopraxia/echolalia and perseveration, $1 / 39$; mitgehen and grasp reflex, 0/39.

Our data shows that only quetiapine (QTP) was used at significantly higher rates at the time of recovery from catatonia $(\mathrm{n}=24,61.5 \%, P<0.001)$ and discharge $(\mathrm{n}=20$,
$51.3 \%, P=0.002)$ than at the time of admission $(\mathrm{n}=7,17.9 \%)$. Similarly, of 29 patients who were not administered ECT, QTP was used at significantly higher rates at the time of recovery from catatonia $(\mathrm{n}=14,48.3 \%, P=0.012)$ than at the time of admission $(\mathrm{n}=5,17.2 \%)$. However, at 12 and 30 months after discharge, the rates had decreased to $38.4 \%$ $(\mathrm{n}=15, P=0.044)$ and $25.6 \%(\mathrm{n}=10, P=0.41)$.

The rate of QTP use was significantly higher in patients with severe stupor than in patients with less severe stupor at the time of recovery from catatonia $(P=0.001)$ and discharge $(P<0.001)$. The tendency was decreasing at 12 and 30 months after discharge ( $P=0.18$ and $P=0.088$ ).

One and two patients developed NMS during treatment with first-generation antipsychotics and risperidone, respectively. Two of these three patients with NMS were treated by combination of medication and ECT, and another patient was treated with high-dose BZP. No patient treated with QTP as first-line treatment developed exacerbation of catatonia or severe extrapyramidal side effects.

The mean dosage ( \pm standard deviation [range]) of QTP was $727( \pm 212[300-1100]) \mathrm{mg} /$ day at the time of recovery from catatonia $(n=24)$. Treatment with dosages of QTP near the approved maximum $(750 \mathrm{mg} /$ day in Japan) was safe under

Table I Clinical data of 39 patients

\begin{tabular}{|c|c|c|c|}
\hline & $\begin{array}{l}\text { With less severe stupor } \\
{[n=28]}\end{array}$ & $\begin{array}{l}\text { With severe stupor } \\
{[n=I I]}\end{array}$ & $P$-value \\
\hline \multicolumn{4}{|l|}{ Characteristics } \\
\hline Age, mean \pm SD [range] & $42.0 \pm 13.8[20-72]$ & $35.9 \pm 14.8[16-55]$ & 0.26 \\
\hline Male/female, n (\%) & $10(35.7) / 18(64.3)$ & $5(45.5) / 6(54.5)$ & 0.82 \\
\hline First episode/relapse, n (\%) & $6(21.4) / 22(78.6)$ & $5(45.5) / 6(54.5)$ & 0.96 \\
\hline BFCRS, mean \pm SD & $|5.57 \pm 7.6|$ & $28.09 \pm 7.54$ & 0.00004 \\
\hline CGI-S of acute phase, mean \pm SD & $6.25 \pm 0.59$ & $6.81 \pm 0.40$ & 0.22 \\
\hline CGI-S of maintenance, mean \pm SD & $2.25 \pm 0.89$ & $2.45 \pm 0.82$ & 0.44 \\
\hline With fever/h-CK/NMS & $1 / 7 / 0$ & $\mid 1 / 11 / 3$ & - \\
\hline Duration of catatonia, median [range] & $7[1-180]$ & $28[3-90]$ & 0.35 \\
\hline BZP, mg daily, mean \pm SD & $2.09 \pm 1.32$ & $3.09 \pm 1.63$ & 0.052 \\
\hline Mood stabilizers (VPA/CBZ/Li), n & II (8/I/2) & $5(4 / 0 / I)$ & 0.76 \\
\hline cECT, n (\%) & $4(14.3)$ & $6(54.5)$ & 0.017 \\
\hline \multicolumn{4}{|c|}{ Antipsychotics chosen, QTP [cECT]/others (RIS, OLZ, other SGA, FGA)/none, n } \\
\hline Admission & $5 / 23(14,4,1,4) / 0$ & $2 / 9(7,0,0,2) / 0$ & 0.65 \\
\hline Recovery from catatonia & $13[4] / 15(9,3,0,3) / 0$ & II $[6] / 0 / 0$ & 0.0015 \\
\hline Discharge & $9 / 19(8,5,0,6) / 0$ & $1 \mathrm{I} / 0 / 0$ & 0.00014 \\
\hline 12 months after discharge & $9 / 19(7,4,2,6) / 0$ & $6 / 4(1,1,2,0) / 1$ & 0.18 \\
\hline 30 months after discharge & $5 / 22(9,6,4,3) / 1$ & $5 / 5(2,1,2,0) / 1$ & 0.088 \\
\hline \multicolumn{4}{|c|}{ Dosages of QTP, mg daily, mean \pm SD [range] } \\
\hline Recovery from catatonia & $754 \pm 247[300-1100]$ & $695 \pm 167[450-900]$ & 0.50 \\
\hline Discharge & $850 \pm 146[600-1100]$ & $632 \pm 206[400-900]$ & 0.013 \\
\hline 12 months after discharge & $439 \pm 162[200-700]$ & $592 \pm 169[400-900]$ & 0.11 \\
\hline 30 months after discharge & $640 \pm 351[300-1200]$ & $615 \pm 49[575-700]$ & 0.88 \\
\hline
\end{tabular}

Abbreviations: BFCRS, Bush-Francis Catatonia Rating Scale; BZP, benzodiazepine dosage (lorazepam equivalent) at time of recovery from catatonia; CBZ, carbamazepine; cECT, co-treatment with electroconvulsive therapy; CGI-S, Clinical Global Impression-Severity; FGA, first-generation antipsychotic; h-CK, serum creatine kinase $>500$ IU/L; Li, lithium carbonate; NMS, neuroleptic malignant syndrome; OLZ, olanzapine; QTP, quetiapine; RIS, risperidone; SD, standard deviation; SGA, second-generation antipsychotic; VPA, sodium valproate. 


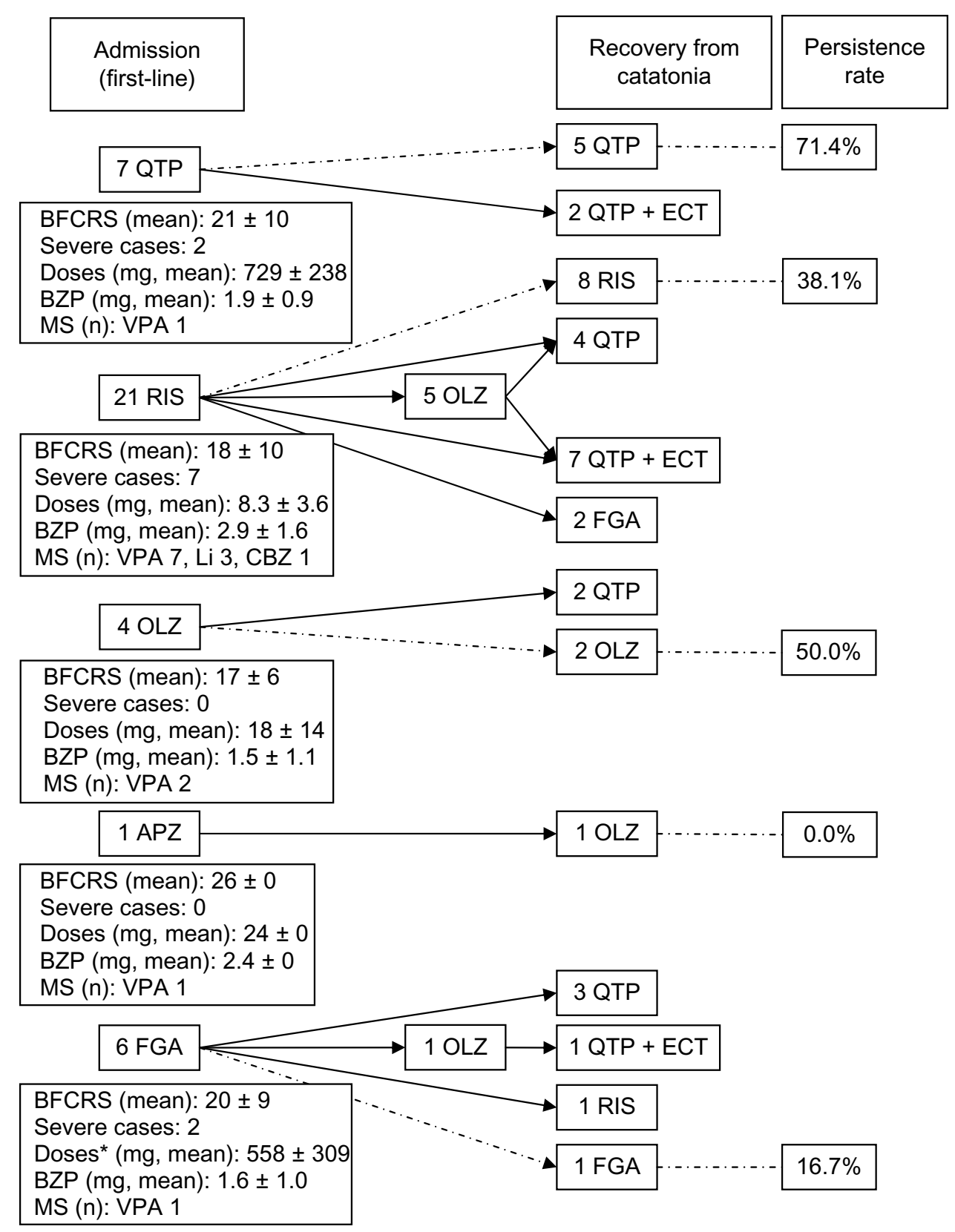

Figure I A flow chart of antipsychotics used for 39 patients during the period from admission to recovery from catatonia.

Note: *Equivalent to chlorpromazine.

Abbreviations: APZ, aripiprazole; BFCRS, Bush-Francis Catatonia Rating Scale; BZP, benzodiazepine dosage (lorazepam equivalent); CBZ, carbamazepine; ECT, electroconvulsive therapy; FGA, first-generation antipsychotic; Li, lithium carbonate; MS, mood stabilizers; OLZ, olanzapine; QTP, quetiapine; RIS, risperidone; VPA, sodium valproate.

conditions in which other antipsychotics are risky. From the time of recovery from catatonia to discharge, the mean dosages of QTP for patients who were administered ECT decreased (from 805 to $733 \mathrm{mg} /$ day), and those for patients who were not administered ECT increased (from 671 to $727 \mathrm{mg} /$ day). Only one patient was treated with high-dose BZP (7.2 mg, equivalent to lorazepam $6 \mathrm{mg}$ or more daily), and 33 patients were treated with low-dose BZP. From the time of recovery from catatonia to 30 months after discharge,
QTP was switched to other antipsychotics for $16 / 24$ patients (insufficient efficacy, eight; sedation, three; hyperglycemia, two; weight gain, one; initiation of depot, two), and other antipsychotics were switched to QTP for 2/15 patients (dysphoria, one; extrapyramidal side effects, one).

\section{Discussion}

To our knowledge, this is the first report of a naturalistic investigation indicating that QTP might be a promising 
agent in the treatment of acute schizophrenia with catatonic stupor. Further, QTP might be more effective than other antipsychotics in the presence of catatonic stupor, fever, and h-CK. Only QTP was used at significantly higher rates at the time of recovery from catatonia and discharge than at the time of admission. The same was true in patients who were not administered ECT. The rate of QTP use at the time of recovery from catatonia and discharge was significantly higher in patients with severe stupor than in patients with less severe stupor. On the other hand, the rates had decreased at 12 and 30 months after discharge. Therefore, QTP was specifically more effective during the acute phase than the maintenance phase in the treatment of schizophrenia with catatonic stupor. However, five limitations apply.

First, the rate of QTP use is not necessarily equivalent to the effectiveness of QTP because our research was performed at a single hospital without a specific treatment algorithm and we investigated the medical records retrospectively. Thus, the possibility of physicians' preferences in drug selection cannot be ruled out. The attending physician might have chosen QTP with the possibility in mind that the underlying causes of catatonia were mood disorders such as bipolar depression, for which QTP is effective and first-line treatment. ${ }^{28}$ Most catatonic patients suffer from mood disorders. ${ }^{5,6}$ If a history of present illness is unclear, the underlying cause of catatonia cannot be confirmed because it is difficult to conduct a structured interview with patients with catatonic stupor. However, all of the physicians at Okayama Psychiatric Medical Center during the period of study did not have a marked predilection for QTP. We most frequently used risperidone as first-line treatment for patients with acute schizophrenia characterized by catatonic stupor in the same way as those without catatonia. Further, we generally chose QTP or olanzapine as second-line treatment if first-line antipsychotics were not efficacious or poorly tolerated. Eventually most patients recovered from catatonia and were discharged with QTP or a combination of QTP and ECT. Moreover, we would like to consider the prescription of antipsychotics of patients with the diagnosis of schizophrenia without catatonia $(n=175)$, who received acute psychiatric hospital treatment between April 2008 and March 2009 at our psychiatric acute inpatient ward. Although the investigation period is different from that of this report, the rate of QTP use for non-catatonic schizophrenia patients was continuously about 10\% (admission, 9.7\%; discharge, 8.0\%; 12 months after discharge, $13.2 \%$ ) in our hospital. ${ }^{29}$

Second, this study is a retrospective chart review and the sample size $(n=39)$ is too small. Third, the effectiveness of BZP combined with mood stabilizers was not considered.
Fourth, a more favorable outcome might have been obtained from high-dose BZP without antipsychotics during catatonia. Only one patient had been treated with high-dose BZP in our case series. High-dose BZP trial remained a first option in the treatment of catatonia whatever the condition associated with. ${ }^{4,30}$ Antipsychotic treatment might be one factor that resulted in the development of catatonia. Fifth, clozapine, which may have beneficial effects for catatonia in psychotic patients, ${ }^{31}$ was not used during the acute phase in this study.

The mean dosages of QTP at discharge for patients with severe stupor was significantly lower than those for patients with less severe stupor, because patients with severe stupor were administered ECT at a significantly higher rate than patients with less severe stupor. From the time of recovery from catatonia to discharge, the dosages of QTP for patients who were administered ECT had decreased to improve the tolerability of medication, and the dosages of QTP for patients who were not administered ECT had increased to treat remaining psychotic symptoms.

Catatonia is thought to be predominantly caused by dopamine hypoactivity, specifically at $\mathrm{D}_{2}$ receptors, which leads to an increase in the release of glutamate in an attempt to increase dopamine activity. ${ }^{32}$ In studies on rat models, QTP was shown to increase the release of dopamine, norepinephrine, and glutamate in the medial prefrontal cortex in a dose-dependent fashion without affecting the release of serotonin or gamma-aminobutyric acid levels in the same area. ${ }^{33}$ BZP potentiates the action of gamma-aminobutyric acid, which can inhibit glutamate hyperactivity. ${ }^{32}$ It is possible that there are combined effects of QTP and BZP in the treatment of catatonia.

To further support the hypoactive $\mathrm{D}_{2}$ receptor theory, several case reports exist that demonstrate high-potency typical antipsychotics either causing or worsening catatonia. ${ }^{31,32}$ The antagonism of $\mathrm{D}_{2}$ receptors by QTP is the weakest of all antipsychotics. The affinity for $\mathrm{D}_{2}$ receptors of QTP is weaker than that of intrinsic dopamine (loose binding), ${ }^{34,35}$ and it is the most rapid to dissociate from $\mathrm{D}_{2}$ receptors (transient blockade). ${ }^{36,37}$ Thus, QTP may be tolerable when patients are at risk of NMS if treated with antipsychotics. However, it is necessary to consider the benefits and risks of QTP because cases of NMS have been reported in conjunction with all antipsychotics including QTP. ${ }^{38}$

Although the efficacy of QTP in the treatment of acute schizophrenia with catatonic stupor cannot be confirmed given the small number of cases, retrospective study design which merely describes physician preference and the concurrent use of ECT and/or BZP, our study indicated that this 
antipsychotic agent may be somewhat better tolerated in patients with catatonic stupor.

\section{Acknowledgments}

No financial support for this study was provided. We thank Drs Suguru Ishizu and Jun Goshima for their assistance in conceiving the idea and methodology of this study and writing the manuscript.

\section{Disclosure}

The authors report no conflicts of interest in this work.

\section{References}

1. Fink M, Taylor MA. Catatonia. A Clinician's Guide to Diagnosis and Treatment. Cambridge, UK, Cambridge University Press, 2003.

2. American Psychiatric Association. Diagnostic and Statistical Manual of Mental Disorders. 4th ed. Washington, DC, American Psychiatric Association, 1994.

3. Bush G, Fink M, Petrides G, Dowling F, Francis A. Catatonia. I. Rating scale and standardized examination. Acta Psychiatr Scand. 1996;93(2): 129-136.

4. Fink M, Shorter E, Taylor MA. Catatonia is not schizophrenia: Kraepelin's error and the need to recognize catatonia as an independent syndrome in medical nomenclature. Schizophr Bull. 2010;36(2): 314-320.

5. Rosebush PI, Mazurek MF. Catatonia and its treatment. Schizophr Bull. 2010;36(2):239-242.

6. Taylor MA, Fink M. Catatonia in psychiatric classification: a home of its own. Am J Psychiatry. 2003;160(7):1233-1241.

7. Ghaziuddin N, Dhossche D, Marcotte K. Retrospective chart review of catatonia in child and adolescent psychiatric patients. Acta Psychiatr Scand. 2012;125(1):33-38.

8. Singerman B, Raheja R. Malignant catatonia - a continuing reality. Ann Clin Psychiatry. 1994;6(4):259-266.

9. White DA. Catatonia and the neuroleptic malignant syndrome - a single entity? Br J Psychiatry. 1992;161:558-560.

10. Carroll BT, Taylor RE. The nondichotomy between lethal catatonia and neuroleptic malignant syndrome. J Clin Psychopharmacol. 1997;17(3): 235-238.

11. Fink M, Taylor MA. The many varieties of catatonia. Eur Arch Psychiatry Clin Neurosci. 2001;251 Suppl 1:I8-I13.

12. Rosebush PI, Mazurek MF. Catatonia: re-awakening to a forgotten disorder. Mov Disord. 1999;14(3):395-397.

13. Ungvari GS, Leung CM, Wong MK, Lau J. Benzodiazepines in the treatment of catatonic syndrome. Acta Psychiatr Scand. 1994;89(4): 285-288.

14. Yassa R, Iskandar H, Lalinec M, Cleto L. Lorazepam as an adjunct in the treatment of catatonic states: an open clinical trial. $J$ Clin Psychopharmacol. 1990;10(1):66-68.

15. Bush G, Fink M, Petrides G, Dowling F, Francis A. Catatonia. II. Treatment with lorazepam and electroconvulsive therapy. Acta Psychiatr Scand. 1996;93(2):137-143.

16. Hawkins JM, Archer KJ, Strakowski SM, Keck PE. Somatic treatment of catatonia. Int J Psychiatry Med. 1995;25(4):345-369.

17. Fink M. Rediscovering catatonia: the biography of a treatable syndrome. Acta Psychiatr Scand Suppl. 2013;127(s441):1-47.

18. Ungvari GS, Chiu HF, Chow LY, Lau BS, Tang WK. Lorazepam for chronic catatonia: a randomized, double-blind, placebo-controlled cross-over study. Psychopharmacology (Berl). 1999;142(4):393-398.
19. Kirino E. Prolonged catatonic stupor successfully treated with aripiprazole in an adolescent male with schizophrenia: a case report. Clin Schizophr Relat Psychoses. 2010;4(3):185-188.

20. Strawn JR, Delgado SV. Successful treatment of catatonia with aripiprazole in an adolescent with psychosis. J Child Adolesc Psychopharmacol. 2007;17(5):733-735.

21. Grenier E, Ryan M, Ko E, Fajardo K, John V. Risperidone and lorazepam concomitant use in clonazepam refractory catatonia: a case report. J Nerv Ment Dis. 2011;199(12):987-988.

22. Valevski A, Loebl T, Keren T, Bodinger L, Weizman A. Response of catatonia to risperidone: two case reports. Clin Neuropharmacol. 2001;24(4):228-231.

23. Babington PW, Spiegel DR. Treatment of catatonia with olanzapine and amantadine. Psychosomatics. 2007;48(6):534-536.

24. Guzman CS, Myung VH, Wang YP. Treatment of periodic catatonia with atypical antipsychotic, olanzapine. Psychiatry Clin Neurosci. 2008;62(4):482.

25. Angelopoulos EK, Corcondilas M, Kollias CT, Kioulos KT, Bergiannaki JD, Papadimitriou GN. A case of catatonia successfully treated with ziprasidone, in a patient with DSM-IV delusional disorder. J Clin Psychopharmacol. 2010;30(6):745-746.

26. Hung YY, Yang PS, Huang TL. Clozapine in schizophrenia patients with recurrent catatonia: report of two cases. Psychiatry Clin Neurosci. 2006;60(2):256-258.

27. Caroff SN, Mann SC. Neuroleptic malignant syndrome and malignant hyperthermia. Anaesth Intensive Care. 1993;21(4):477-478.

28. Vieta E, Valentí M. Pharmacological management of bipolar depression: acute treatment, maintenance, and prophylaxis. CNS Drugs. 2013;27(7): 515-529.

29. Takaki M, Yoshimura B, Kono T, et al. [Treatment of schizophrenia at emergency unit in Okayama Psychiatric Medical Center, Japan: Effectiveness and selection of antipsychotics at acute and maintenance, intervention and prognosis]. Jpn J Clin Psychopharmacol. 2010;13: 943-955. Japanese.

30. Lin CC, Huang TL. Lorazepam-diazepam protocol for catatonia in schizophrenia: A 21-case analysis. Compr Psychiatry. 2013.

31. England ML, Ongür D, Konopaske GT, Karmacharya R. Catatonia in psychotic patients: clinical features and treatment response. J Neuropsychiatry Clin Neurosci. 2011;23(2):223-226.

32. Carroll BT, Lee JWY, Appiani F, Thomas C. The Pharmacotherapy of Catatonia. Primary Psychiatry. 2010;17(4):41-47.

33. Yamamura $\mathrm{S}$, Ohoyama $\mathrm{K}$, Hamaguchi $\mathrm{T}$, et al. Effects of quetiapine on monoamine, GABA, and glutamate release in rat prefrontal cortex. Psychopharmacology (Berl). 2009;206(2):243-258.

34. Richelson E, Souder T. Binding of antipsychotic drugs to human brain receptors focus on newer generation compounds. Life Sci. 2000;68(1): 29-39.

35. Kasper S, Tauscher J, Küfferle B, Barnas C, Pezawas L, Quiner S. Dopamine- and serotonin-receptors in schizophrenia: results of imagingstudies and implications for pharmacotherapy in schizophrenia. Eur Arch Psychiatry Clin Neurosci. 1999;249 Suppl 4:83-89.

36. Takano A, Suhara T, Ikoma Y, et al. Estimation of the time-course of dopamine D2 receptor occupancy in living human brain from plasma pharmacokinetics of antipsychotics. Int $J$ Neuropsychopharmacol. 2004;7(1):19-26.

37. Kapur S, Seeman P. Does fast dissociation from the dopamine $\mathrm{d}(2)$ receptor explain the action of atypical antipsychotics?: A new hypothesis. Am J Psychiatry. 2001;158(3):360-369.

38. El-Gaaly S, St John P, Dunsmore S, Bolton JM. Atypical neuroleptic malignant syndrome with quetiapine: a case report and review of the literature. J Clin Psychopharmacol. 2009;29(5):497-499. 
Neuropsychiatric Disease and Treatment

Dovepress

\section{Publish your work in this journal}

Neuropsychiatric Disease and Treatment is an international, peerreviewed journal of clinical therapeutics and pharmacology focusing on concise rapid reporting of clinical or pre-clinical studies on a range of neuropsychiatric and neurological disorders. This journa is indexed on PubMed Central, the 'PsycINFO' database and CAS

The manuscript management system is completely online and includes a very quick and fair peer-review system, which is all easy to use. Visit http://www.dovepress.com/testimonials.php to read real quotes from published authors.

Submit your manuscript here: http://www.dovepress.com/neuropsychiatric-disease-and-treatment-journal 